\title{
Symmetry Transformations from Local Currents
}

\author{
H. DieTER MaISON \\ Max-Planck-Institut für Physik und Astrophysik, München
}

Received February 2, 1969

\begin{abstract}
For internal symmetries it is shown that it is possible to construct automorphisms for a Haag-Araki local ring system $\{\mathscr{R}(\mathcal{O})\}$ from a local current affiliated to it. Although the "charges" $Q_{V}$ for finite volume $V$ do not converge for $V \rightarrow \infty$ we prove the convergence of the corresponding automorphisms of $\{\mathscr{R}(\mathcal{O})\}$. For external symmetries which map bounded space-time regions into unbounded ones (e.g. translations) we have to require some additional continuity condition on the isomorphisms corresponding to $Q_{V}$ to get convergence.
\end{abstract}

In the usual Lagrangian formulation of Quantum Field Theory one derives in a formal way a local current $j_{G}^{\mu}($.$) for every one-para-$ meter transformation group $G$ which acts nontrivially on the fields. Formally the space-integral $\int j_{G}^{0}(x, t) d^{3} x$ serves as infinitesimal generator for a unitary representation of $G$ in the Hilbertspace of states. However, because of vacuum fluctuations, the local"charges" $Q_{V}(t)=\int_{V} j^{0}(x, t) d^{3} \boldsymbol{x}$ for finite volume $V$ turn out not to converge in any useful way (strong or weak topology for operators) for increasing volume [1], Theorem 3.1, even if one takes care of distributional difficulties and smears the current in space and time with $C_{0}^{\infty}$-functions. So the question arises how to construct symmetry transformations for the algebra of fields or observables from a given local current $j^{\mu}$. This problem also arises in the usual formulation of the "Goldstone Theorem" $[1,2]$ where one assumes the existence of a group of automorphisms of the algebra of quasilocal observables generated by a local current $j^{\mu}$. One may ask then if these assumptions are compatible.

Since one is not primarily interested in a global unitary transformation to implement the symmetry, which may not even exist as in the case of spontaneously broken symmetries, it would be sufficient if the local symmetry transformations $\alpha_{V}(\tau) A=e^{i \tau Q_{V}} A e^{-i \tau Q_{V}}$ for the algebra of fields or observables $\mathscr{R}(\mathcal{O})$ from some bounded space-time region $\mathcal{O}$ would converge with increasing volume $V$. This problem is studied in the framework of local v. Neumann algebras in the HaagAraki [3] sense. In Section 1 we provide some mathematical tools 
giving the connection between the generator $Q$ of a unitary group $\mathscr{U}(\tau)=e^{i \tau Q}$ in a Hilbert space $H$ and the generator of the corresponding group of automorphisms $\alpha(\tau) A=\mathscr{U}(\tau) A \mathscr{U}^{-1}(\tau)$ of the algebra of bounded operators $\mathscr{B}(H)$ equipped with several interesting topologies.

In Section 2 we give a solution of the problem mentioned above for internal symmetries under rather natural assumptions. In Section 3 we consider the case of space-time symmetries and give a solution under the further assumption (not very natural from a field-theoretic view-point) that the local automorphisms $\alpha_{V}(\tau)$ are strongly continuous in $\tau$ in the uniform operator topology on the local algebras $\mathfrak{A}(\mathcal{O})$.

\section{On Generators of Unitarily Implemented Automorphism Groups}

Throughout this section $Q$ is assumed to be an essentially selfadjoint (e.s.a.) operator on some domain $D(Q)$ dense in a Hilbert space $H$. $\mathscr{B}(H)$ denotes the algebra of bounded operators on $H$. Then $Q^{*}$ is the uniquely determined self-adjoint extension of $Q$ in $H$. It is the generator of a strongly continuous group of unitaries $\mathscr{U}(\tau)=e^{i \tau Q^{*}}$ which gives rise to a one-parameter group of automorphisms $\alpha(\tau) A=\mathscr{U}(\tau) A \mathscr{U}^{-1}(\tau)$ of $\mathscr{B}(H) . \alpha() A.(A \in \mathscr{B}(H))$ is a continuous map of $\boldsymbol{R}^{1} \rightarrow \mathscr{B}(H)$ equipped with the strong or weak topology from vectors in $H^{1}$, but not in general with the norm topology of operators on $\mathscr{B}(H)$. For fixed $\tau, \alpha(\tau)$ is a continuous map of $\mathscr{B}(H) \rightarrow \mathscr{B}(H)$ for all these topologies. The family $\{\alpha(\tau)\}_{\tau \in \boldsymbol{R}^{\mathbf{1}}}$ is an equicontinuous set in general only for the norm topology on $\mathscr{B}(H)$.

Lemma 1. $Q$ e.s.a. on $D(Q), A \in \mathscr{B}(H) \Rightarrow$

$$
\frac{d}{d \tau}(x, \alpha(\tau) A y)=i\left(Q^{*} x, \alpha(\tau) A y\right)-i\left(x, \alpha(\tau) A Q^{*} y\right) \forall x, y \in D\left(Q^{*}\right) .
$$

Proof. Lemma 1 is an immediate consequence of Stone's theorem [4].

Lemma 2. $Q$ e.s.a. on $D(Q), A \in \mathscr{B}(H), A D\left(Q^{*}\right) \subset D\left(Q^{*}\right) \Rightarrow$

$$
\frac{d \alpha(\tau)}{d \tau} A x=s \cdot \lim _{h \rightarrow 0} \frac{\alpha(\tau+h)-\alpha(\tau)}{h} A x=i\left[Q^{*}, \alpha(\tau) A\right] x, \forall x \in D\left(Q^{*}\right) \text {. }
$$

Proof.

$$
\begin{aligned}
& \left\|\left(\frac{\alpha(\tau+h)-\alpha(\tau)}{h} A-i\left[Q^{*}, \alpha(\tau) A\right]\right) x\right\| \\
\leqq & \left\|\left(\frac{\mathscr{U}(\tau+h)-\mathscr{U}(\tau)}{h} A \mathscr{U}^{*}(\tau)-i Q^{*} \mathscr{U}(\tau) A \mathscr{U}^{*}(\tau)\right) x\right\|
\end{aligned}
$$

\footnotetext{
${ }^{1}$ In the following "weak" is always to be interpreted in this sense.
} 


$$
\begin{aligned}
& +\left\|\left(\mathscr{U}(\tau+h) A \frac{\mathscr{U}^{*}(\tau+h)-\mathscr{U}^{*}(\tau)}{h}+i \mathscr{U}(\tau+h) A \mathscr{U}^{*}(\tau) Q^{*}\right) x\right\| \\
& +\left\|(\mathscr{U}(\tau)-\mathscr{U}(\tau+h)) A \mathscr{U}^{*}(\tau) Q^{*} x\right\| \\
& =\left\|\left(\frac{\mathscr{U}(h)-1}{h} \mathscr{U}(\tau) A \mathscr{U}^{*}(\tau)-i Q^{*} \mathscr{U}(\tau) A \mathscr{U}^{*}(\tau)\right) x\right\| \\
& +\left\|\left(\mathscr{U}(\tau) A \mathscr{U}^{*}(\tau) \frac{\mathscr{U}^{*}(h)-1}{h}+i \mathscr{U}(\tau) A \mathscr{U}^{*}(\tau) Q^{*}\right) x\right\| \\
& +\left\|(1-\mathscr{U}(h)) A \mathscr{U}^{*}(\tau) Q^{*} x\right\|
\end{aligned}
$$

which tends to zero for $h \rightarrow 0$ for all $x \in D\left(Q^{*}\right)$.

Lemma 3. $Q$ e.s.a. on $D(Q), A \in \mathscr{B}(H), A D(Q) \subset D\left(Q^{*}\right)$,

$$
\left\|\left[Q^{*}, A\right] x\right\| \leqq c\|x\|, \quad \forall x \in D(Q) \Rightarrow A D\left(Q^{*}\right) \subset C D\left(Q^{*}\right) .
$$

Proof. Let $x \in D\left(Q^{*}\right)$ arbitrary, then there exists a sequence $x_{n} \in D(Q)$ with $x_{n} \underset{n \rightarrow \infty}{\longrightarrow} x$ and $Q x_{n} \longrightarrow{ }_{n \rightarrow \infty} Q^{*} x$ since $Q^{*}$ is the closure of $Q$. We derive $Q^{*} A x_{n}=A Q x_{n}+\left[Q^{*}, A\right] x_{n} \underset{n \rightarrow \infty}{\longrightarrow} A Q^{*} x+\left[Q^{*}, A\right]^{-} x^{2}$. From $Q^{*}$ being closed we get $A x \in D\left(Q^{*}\right)$.

Lemma 4. For a map $u($.$) from \boldsymbol{R}^{1}$ into a Banachspace $X$ the following statements are equivalent:

i) $u($.$) is analytic at t=0$.

ii) $u($.$) is infinitely differentiable in some neighbourhood |t|<\delta$ of $t=0$ and there exist $M>0, a>0$ with $\left\|u^{(n)}(t)\right\| \leqq M n ! a^{n}$ for all $|t|<\delta$ and $n \in N$.

Proof. i) $\Rightarrow$ ii): $u($.$) may be continued to a holomorphic function$ $\tilde{u}($.$) in some disk |z|<R$ and we get

$$
\tilde{u}^{(n)}(z)=\frac{n !}{2 \pi i} \int_{|\zeta|=R / 2} \frac{\tilde{u}(\zeta) d \zeta}{(\zeta-z)^{n+1}}, \quad \forall|z|<R / 2 .
$$

If we set $M=\sup _{|z|=R / 2}\|\tilde{u}(z)\|$ and $a=2 / R$ we get the desired estimate for $u^{(n)}(t)$ in $|t|<R / 2$.

$$
\text { ii) } \Rightarrow \text { i): } \quad \begin{aligned}
u(t) & =\sum_{k=0}^{n-1} \frac{u^{(k)}(0)}{k !} t^{k}+\int_{0}^{1} \frac{t^{n}(1-\tau)^{n-1}}{(n-1) !} u^{(u)}(t \tau) d \tau, \quad \forall n \in N \\
& \Rightarrow\left\|u(t)-\sum_{k=0}^{n-1} \frac{u^{(u)}(0)}{t !} t^{k}\right\| \leqq M(a t)^{n} \underset{n \rightarrow \infty}{\longrightarrow} 0 \text { for } \quad|t|<1 / a .
\end{aligned}
$$

\footnotetext{
${ }^{2} B^{-}$denotes the closure of $B$.
} 
Now we are able to prove some propositions which give us the announced connection between the infinitesimal properties of $\alpha($.$) and \mathscr{U}($.$) :$

Proposition 1. Qe.s.a. on $D(Q), A \in \mathscr{B}(H), \alpha(\tau) A=e^{i \tau Q^{*}} A e^{-i \tau Q^{*}}$ then the following statements are equivalent:

i) $A D(Q) \subset D\left(Q^{*}\right), \|$ ad $Q^{*} A x\|\leqq c\| x \|, \forall x \in D(Q),{ }^{3}$

ii) $\alpha() A:. \boldsymbol{R}^{1} \rightarrow \mathscr{B}(H)$ is weakly differentiable ${ }^{4}$,

iii) $\alpha() A:. \boldsymbol{R}^{1} \rightarrow \mathscr{B}(H)$ is strongly differentiable, and under the extra assumption that $\alpha() a. d Q^{*} A$ is continuous in the uniform (norm) topology on $\mathscr{B}(H)$,

iv) $\alpha() A:. \boldsymbol{R}^{1} \rightarrow \mathscr{B}(H)$ is differentiable in norm; from i)-iv) it follows

$$
\frac{d \alpha(\tau)}{d \tau} A=i\left(\operatorname{ad} Q^{*} \alpha(\tau) A\right)^{-}=i \alpha(\tau)\left(\operatorname{ad} Q^{*} A\right)^{-} .
$$

Proof. iv) $\Rightarrow$ iii) $\Rightarrow$ ii) are trivial.

ii) $\Rightarrow$ i): From Lemma 1 we know

$$
\begin{aligned}
& \frac{d}{d \tau}(x, \alpha(\tau) A y)=i(Q x, \alpha(\tau) A y)-i(x, \alpha(\tau) A Q y), \quad \forall x, y \in D(Q) \\
& \quad \Rightarrow|(Q x, \alpha(\tau) A y)| \leqq|(x, \alpha(\tau) A Q y)|+\left|\left(x, \frac{d \alpha(\tau)}{d \tau} A y\right)\right| \\
& \quad \leqq\|x\|\left(\|\alpha(\tau) A Q y\|+\left\|\frac{d \alpha(\tau)}{d \tau} A y\right\|\right), \quad \forall x, y \in D(Q) \Rightarrow \\
& \quad \alpha(\tau) A y \in D\left(Q^{*}\right)
\end{aligned}
$$

for all $y \in D(Q)$ according to the Riesz representation theorem. So we have $\alpha(\tau) A D(Q) \subset D\left(Q^{*}\right)$ and from Eq. (1.1) we deduce $\frac{d \alpha(\tau)}{d \tau} A=i\left(\operatorname{ad} Q^{*} \alpha(\tau) A\right)^{-}=i \alpha(\tau)\left(\operatorname{ad} Q^{*} A\right)^{-}$because $\left[\mathscr{U}(\tau), Q^{*}\right] \leqq 0$.

So we get $\left\|\operatorname{ad} Q^{*} A x\right\| \leqq\|\delta A\|\|x\|, \forall x \in D\left(Q^{*}\right)$ with the definition

$$
\delta A:=\left.\frac{d \alpha(\tau)}{d \tau} A\right|_{\tau=0} \cdot{ }^{4}
$$

i) $\Rightarrow$ iii): From Lemma 3 we have $A D\left(Q^{*}\right) \subset D\left(Q^{*}\right)$ so we can apply Lemma 2 to get

$$
\frac{d \alpha(\tau)}{d \tau} A x=i \operatorname{ad} Q^{*} \alpha(\tau) A x, \quad \forall x \in D\left(Q^{*}\right) .
$$

${ }^{3}$ ad $Q^{*} A$ denotes $\left[Q^{*}, A\right]$, inductively $\left(\operatorname{ad} Q^{*}\right)^{n} A=\left[Q^{*},\left(\operatorname{ad} Q^{*}\right)^{n-1} A\right]$.

${ }^{4}$ Differentiability means existence of the limit $\lim _{h \rightarrow 0} \frac{\alpha(\tau+h)-\alpha(\tau)}{h} A$ in $\mathscr{B}(H)$. 
Using the identity

$$
\frac{\alpha(h)-1}{h} A x=\int_{0}^{1} \frac{d \alpha(t h)}{d t} A x d t=i \int_{0}^{1} \operatorname{ad} Q^{*} \alpha(t h) A x d t, \quad \forall x \in D\left(Q^{*}\right)
$$

we arrive at

$$
\begin{aligned}
\left\|\frac{\alpha(h)-1}{h} A x\right\| & \leqq \int_{0}^{1}\left\|\alpha(t h) \operatorname{ad} Q^{*} A x\right\| d t \leqq\left\|\operatorname{ad} Q^{*} A\right\|\|x\|, \quad \forall x \in D\left(Q^{*}\right) \\
& \Rightarrow\left\|\frac{\alpha(h)-1}{h} A\right\| \leqq\left\|\operatorname{ad} Q^{*} A\right\| \leqq C .
\end{aligned}
$$

Consequently the family $\{1 / h(\alpha(\tau+h)-\alpha(\tau)) A\}_{h \in \boldsymbol{R}^{1}}$ of bounded operators on $H$ is equi-bounded since $\|\alpha(\tau) A\| \leqq\|A\|$, converging strongly on the dense set $D\left(Q^{*}\right)$ for $h \rightarrow 0$. Thus it converges strongly on all of $H$ and

$$
\begin{aligned}
& \frac{d \alpha(\tau)}{d \tau} A x=i\left(\operatorname{ad} Q^{*} \alpha(\tau) A\right)^{-} x, \quad \forall x \in H . \\
\text { iii) } \Rightarrow \text { iv }): & \\
\left\|\left(\frac{\alpha(\tau+h)-\alpha(\tau)}{h} A-i \alpha(\tau) \operatorname{ad} Q^{*} A\right) x\right\| & \left\|\int_{0}^{1}(\alpha(t h+\tau)-\alpha(\tau)) \operatorname{ad} Q^{*} A x d t\right\| \\
\leqq & \sup _{|t| \leqq 1}\left\|(\alpha(t h+\tau)-\alpha(\tau)) \operatorname{ad} Q^{*} A\right\|\|x\| \\
\Rightarrow & \| \frac{\alpha(\tau+h)-\alpha(\tau)}{h} A-i \alpha(\tau) \text { ad } Q^{*} A \| \\
\leqq & \sup _{|t| \leqq 1} \|(\alpha(\tau+t h)-\alpha(\tau)) \text { ad } Q^{*} A \| \underset{h \rightarrow 0}{\Rightarrow} 0 ; \\
\Rightarrow & \text { iv) using the assumed continuity of } \alpha(.) \operatorname{ad} Q^{*} A \text { in norm. }
\end{aligned}
$$

Corollary. For $k \in N, 1 \leqq k \leqq \infty$ the following statements are equivalent :

i) $\left(\operatorname{ad} Q^{*}\right)^{n-1} A D(Q) \subset D\left(Q^{*}\right),\left\|\left(\operatorname{ad} Q^{*}\right)^{n} A x\right\| \leqq c_{n}\|x\|, \forall x \in D(Q)$, $1 \leqq n \leqq k$,

ii) $\alpha() A:. \boldsymbol{R}^{1} \rightarrow \mathscr{B}(H)$ is $k$-times weakly differentiable,

iii) $\alpha() A:. \boldsymbol{R}^{1} \rightarrow \mathscr{B}(H)$ is $k$-times strongly differentiable, and under the extra assumption that $\alpha(.)\left(\operatorname{ad} Q^{*}\right)^{n} A$ is continuous in the norm topology of $\mathscr{B}(H)$ for $1 \leqq n \leqq k$,

iv) $\alpha() A:. \boldsymbol{R}^{1} \rightarrow \mathscr{B}(H)$ is $k$-times differentiable in norm.

i)-iv) $\Rightarrow \alpha^{(n)}(\tau) A=\alpha(\tau)\left(\left(i \operatorname{ad} Q^{*}\right)^{n} A\right)^{-}, 1 \leqq n \leqq k$. 
Proof. By induction using Proposition 1.

Proposition 2. If $Q$ is e.s.a. and $Q \in \mathscr{B}(H)$ the following statements are equivalent:

i) $\left(\operatorname{ad} Q^{*}\right)^{n-1} A D(Q) \subset D\left(Q^{*}\right)$ and there exist $M>0, a>0$ with $\left\|\left(\operatorname{ad} Q^{*}\right)^{n} A x\right\| \leqq M n ! a^{n}\|x\|, \forall x \in D(Q), n \in N$,

ii) $\alpha() A:. \boldsymbol{R}^{1} \rightarrow \mathscr{B}(H)$ is weakly analytic,

iii) $\alpha() A:. \boldsymbol{R}^{1} \rightarrow \mathscr{B}(H)$ is strongly analytic,

iv) $\alpha() A:. \boldsymbol{R}^{1} \rightarrow \mathscr{B}(H)$ is analytic in norm.

Proof. iv) $\Rightarrow$ iii) $\Rightarrow$ ii) are trivial.

iv) $\Rightarrow$ i): From $\alpha()$.$A being infinitely differentiable we get$

$\left(\operatorname{ad} Q^{*}\right)^{n} A D(Q) \subset D\left(Q^{*}\right), \alpha^{(n)}(\tau) A x=\left(\left(i \operatorname{ad} Q^{*}\right)^{n} \alpha(\tau) A\right)^{-} x, \forall x \in H, n \in N$.

Lemma 4 gives the existence of $M>0$ and $a>0$ with

$$
\left\|\alpha^{(n)}(0) A\right\|=\left\|\left(\operatorname{ad} Q^{*}\right)^{n} A\right\| \leqq M n ! a^{n}, \quad \forall n \in N .
$$

i) $\Rightarrow$ ii): Firstly we notice $\alpha()$.$A being infinitely often weakly dif-$ ferentiable and $\left|\left(x, \alpha^{(n)}(\tau) A y\right)\right|=\left|\left(x, \alpha(\tau)\left(\operatorname{ad} Q^{*}\right)^{n} A y\right)\right| \leqq\|x\|\|y\| M n ! a^{n}$ for all $\tau \in \boldsymbol{R}^{1}, n \in \boldsymbol{N}$. It follows that $(x, \alpha(\tau) A y)$ is analytic for all $x, y \in H$ using again Lemma 4 for $X=C$.

ii) $\Rightarrow$ iii) (compare [7], p. 52, Lemma 3): $\sum_{n=0}^{\infty}\left(\operatorname{ad} Q^{*}\right)^{n} A y|\tau|^{n} / \mathrm{n} !$ converges weakly for $|\tau|<1 / a$, so $\left\{\left\|\left(\operatorname{ad} Q^{*}\right)^{n} A y\right\||\tau|^{n} / n !\right\}_{n \in N}$ is bounded for all $y \in H$. We choose $\varepsilon>0$ with

$$
\begin{aligned}
(1+\varepsilon)|\tau| & <1 / a \Rightarrow \sum_{n=0}^{\infty}\left\|\left(\operatorname{ad} Q^{*}\right)^{n} A y\right\||\tau| / n ! \\
& =\sum_{n=0}^{\infty}\left\|\left(\operatorname{ad} Q^{*}\right)^{n} A y\right\|((1+\varepsilon)|\tau|)^{n}(n !)^{-1}(1+\varepsilon)^{-n} \leqq C \sum_{n=0}^{\infty}(1+\varepsilon)^{-n} .
\end{aligned}
$$

iii) $\Rightarrow$ iv): For $y \in H$ there exists $M(y)$ with $\left\|\alpha^{(n)}(0) A y\right\| \leqq n ! a^{n} M(y)$ by Lemma 4 . So we get

$$
\|\alpha(\tau) A y\| \leqq \sum_{n=0}^{\infty}\left\|\left(\operatorname{ad} Q^{*}\right)^{n} A y\right\||\tau|^{n} / n ! \leqq M(y)(1-\alpha|\tau|)^{-1} .
$$

By the uniform-boundedness principle we obtain $\|\left(1-a|\tau| \alpha(\tau) A \| \leqq C^{\prime}\right.$. Now we can apply a known theorem [5], p. 365 giving the desired result.

Remark. As the reader may have already noticed there is no extra condition for concluding iv) from i)-iii) in this case. 


\section{Internal Symmetries}

For the following we assume that we are given a local ring system in the Haag-Araki sense [3], the $\mathscr{R}(\mathcal{O})$ are assumed to be v. Neumann algebras of operators on some Hilbertspace $H$. We consider a local current $j^{\mu}$ affiliated to $\{\mathscr{R}(\mathcal{O})\}$. From $j^{\mu}$ we construct local "charge" operators $Q_{r, \alpha}[1]$ by $Q_{r, \alpha}=j^{0}\left(f_{r} \otimes \alpha\right)$ with $f_{r} \in C_{0}^{\infty}\left(\boldsymbol{R}^{3}\right), \alpha \in C_{0}^{\infty}\left(\boldsymbol{R}^{1}\right)$

$$
f_{r}(x)=\left\{\begin{array}{lll}
1 & \text { for } & |x| \leqq r \\
0 & \text { for } & |x| \geqq r+1
\end{array}\right\}, \quad \int \alpha(t) d t=1 .
$$

The charges $Q_{r}$ (we keep $\alpha$ fixed and suppress the index $\alpha$ from now on) are assumed essentially self-adjoint on some common domain $D \subset H$ giving rise to automorphisms $\alpha_{r}(\tau) A=e^{i \tau Q_{r}^{*}} A e^{-i \tau Q^{*}}$ of $\mathscr{B}(H)$. From the relative locality of $j^{\mu}$ with respect to $\mathscr{R}(\mathcal{O})$ we deduce for bounded $\mathcal{O}$ the existence of $r_{0}$ such that

$\left(Q_{r} x, A y\right)-\left(x, A Q_{r} y\right)=\left(Q_{r}, x, A y\right)-\left(x, A Q_{r}, y\right)$ for $r, r^{\prime} \geqq r_{0}$, $\forall A \in \mathscr{R}(\mathcal{O}), x, y \in D$.

For the definition of an internal symmetry we follow Ref. [6]:

Definition. A symmetry is called "internal" if $\alpha_{r}(\tau) \mathscr{R}(\mathcal{O}) \subset \mathscr{R}(\mathcal{O})$, $\forall \tau \in \boldsymbol{R}^{1}$ and $r$ sufficiently big.

A symmetry which is not internal we call "external".

Our statement now is that for internal symmetries and bounded $\mathcal{O}$ the restrictions $\alpha_{r}() \mid. \mathscr{R}(\mathcal{O})$ of $\alpha_{r}($.$) to \mathscr{R}(\mathcal{O})$ all coincide for sufficiently big $r$, thus $\lim _{r \rightarrow \infty} \alpha_{r}() \mid. \mathscr{R}(\mathcal{O})$ exists trivially. $D \subset H$

Theorem 1. Let $Q_{r}$ be essentially self-adjoint on a common domain

$$
\alpha_{r}(\tau) A=e^{-i \tau Q_{r}^{*}} A e^{-i \tau Q_{r}^{*} \in \mathscr{R}(\mathcal{O})} \text { for } A \in \mathscr{R}(\mathcal{O}), r \geqq r_{0}
$$

and Eq. (2.1) for, $r \geqq r_{0}, x, y \in D, A \in \mathscr{R}(\mathcal{O})$, then $\alpha_{r}(\tau) A=\alpha_{r^{\prime}}(\tau) A, \forall A \in \mathscr{R}(\mathcal{O})$, $\tau \in \boldsymbol{R}^{1}, r, r^{\prime} \geqq r_{0}$.

Proof. We consider $\mathscr{R}(\mathcal{O})$ equipped with the weak topology from vectors of $H$ as a quasicomplete locally convex topological vector space ${ }^{5}$; then $\alpha_{r}()$.$A is a continuous map from R^{1}$ into $\mathscr{R}(\mathcal{O})$ for all $A \in \mathscr{R}(\mathcal{O})$. All elements of $\mathscr{R}(\mathcal{O})$ are weakly exponential ${ }^{6}$ vectors for $\alpha_{r}($.$) since$ $|(x, \alpha(\tau) A y)| \in\|A\|\|x\|\|y\|$. So we can apply a generalization of a theorem of Gårding to quasicomplete locally convex topological vector spaces [7]

${ }^{5}$ The topology of $\mathscr{R}(\mathcal{O})$ is defined by the family of seminorms $p(A)=\sum_{k=1}^{n}\left|\left(x_{k}, A y_{k}\right)\right|$ with $x_{k}, y_{k} \in H$ arbitrary.

6 A vector $A$ is called weakly exponential for $\alpha($.) if for any continuous linear functional $\varphi$ on $\mathscr{R}(\mathcal{O})$ there exist constants $a>0$ and $b>0$ with $\varphi(\alpha(\tau) A) \leqq a e^{b|\tau|}$. See Ref. [7]. 
which asserts the existence of a dense supply of analytic vectors for each $\alpha_{r}\left(\right.$.) which we denote by $C_{r}^{\omega}$. We want to show that $C_{r}^{\omega}=C_{r^{\prime}}^{\omega}$ for $r, r^{\prime} \geqq r_{0}$ Assume therefore $A \in C_{r}^{\omega}$ then $\left(\operatorname{ad} Q_{r}^{*}\right)^{n} A D \subset D\left(Q_{r}^{*}\right)$ and there exist $M_{r}>0, \alpha_{r}>0$ with $\left\|\left(\operatorname{ad} Q_{r}^{*}\right)^{n} A\right\| \leqq M_{r} n ! a_{r}^{n}, \forall n \in N$ according to Proposition 2. From Eq. (2.1) we get

$\left|\left(Q_{r^{\prime}} x, A y\right)\right| \leqq\left|\left(x, A Q_{r^{\prime}} y\right)\right|+\left|\left(x, \operatorname{ad} Q_{r}^{*} A y\right)\right| \leqq\|x\|\left(\left\|A Q_{r^{\prime}} y\right\|+\left\|\operatorname{ad} Q_{r}^{*} A y\right\|\right)$

for $\forall x, y \in D$ i.e. $A D \subset D\left(Q_{r^{\prime}}^{*}\right)$. Again from (2.1) we deduce $\left(\operatorname{ad} Q_{r}^{*} A\right)^{-}$ $=\left(\operatorname{ad} Q_{r^{\prime}}^{*} A\right)^{-}$for $r, r^{\prime} \geqq r_{0}$. Repeating this argument we find $\left(\operatorname{ad} Q_{r^{\prime}}^{*}\right)^{n} A D$ $C D\left(Q_{r^{\prime}}^{*}\right)$ and $\left(\left(\operatorname{ad} Q_{r}^{*}\right)^{n} A\right)^{-}=\left(\left(\operatorname{ad} Q_{r^{\prime}}^{*}\right)^{n} A\right)^{-}$for $\forall n \in N$. Therefore $\left\|\left(\operatorname{ad} Q_{r^{\prime}}^{*}\right)^{n} A\right\|$ $\leqq M_{r} n ! a_{r}^{n}$ i.e. $A \in C_{r^{\prime}}^{\omega}$. Thus we have $C_{r}^{\omega} \subset C_{r^{\prime}}^{\omega}$; starting with $C_{r^{\prime}}^{\omega}$ we arrive at $C_{r^{\prime}}^{\omega} \subset C_{r}^{\omega}$, so we have proved $C_{r}^{\omega}=C_{r^{\prime}}^{\omega}$, for $r, r^{\prime} \geqq r_{0}$. Furtheron we have shown

Thus

$$
\alpha_{r}^{(n)}(0) A=\left(i \operatorname{ad} Q_{r}^{*}\right)^{n} A^{-}=\alpha_{r^{\prime}}^{(n)}(0) A \text { for } n \in N, A \in C_{r}^{\omega} .
$$

$$
\alpha_{r}(\tau) A=\sum_{n=0}^{\infty} \frac{\alpha_{r}^{(n)}(0)}{n !} A \tau^{n}=\alpha_{r^{\prime}}(\tau) A \quad \text { for } \quad A \in C_{r}^{\omega}, r, r^{\prime} \geqq r_{0} .
$$

Since the $C_{r}^{\omega}$ lie dense in $\mathscr{R}(\mathcal{O})$ and the $\alpha_{r}(\tau)$ are continuous maps of $\mathscr{R}(\mathcal{O}) \rightarrow \mathscr{R}(\mathcal{O})$ we may extend this equality to all of $\mathscr{R}(\mathcal{O})$.

Remark 1 . We notice that all we need to prove Theorem 1 is a weakly closed subspace of $\mathscr{B}(H)$ which fulfills condition (2.1) for sufficiently big $r$ and $r^{\prime}$. So, if there exists a bounded $\mathcal{O}_{1}$ such that $\left(\bigcup_{\tau \in R^{1}} \alpha_{r}(\tau) \mathscr{R}(\mathcal{O})\right)^{\prime \prime}$ $\subset \mathscr{R}\left(\mathcal{O}_{1}\right)$ for big $r$ and $r^{\prime}$, the assumptions of Theorem 1 hold.

Remark 2. It would be desirable to have some sufficient condition on the $Q_{r}$ that reveals the fact that they give rise to an internal symmetry. The condition ad $Q_{r}^{*} A \in \mathscr{R}(\mathcal{O})$ for a dense set of $A \in \mathscr{R}(\mathcal{O})$ is clearly not sufficient.

\section{External Symmetries}

From Remark 1 to Theorem 1 we conclude that there is no problem with space rotations but only with translations and pure Lorentzrotations. The construction of the global automorphism $\alpha(\tau)=\lim _{r \rightarrow \infty} \alpha_{r}(\tau)$ from local "charges" relies on the equality of the corresponding infinitesimal generators ad $Q_{r}^{*}$ for big $r$. At first sight one may have the impression that it should work equally well for external symmetries since only infinitesimal neighbourhoods of a given bounded region $\mathcal{O}$ seem to be involved. Unfortunately we have used analytic vectors which are generally constructed by smoothing $\alpha_{r}($.$) with analytic functions:$ $A_{f}=\int f(\tau) \alpha_{r}(\tau) A d \tau$ ( $f$ analytic). These $A_{f}$ do generally not belong to 
any $\mathscr{R}\left(\mathcal{O}_{1}\right)$ with bounded $\mathcal{O}_{1}$ if the symmetry changes the region $\mathcal{O}$. So we do not have a dense supply of analytic vectors in the local algebras $\mathscr{R}(\mathcal{O})$ to integrate up the equality of the generators of the $\alpha_{r}$. What we thus need is another method of reconstructing the $\alpha_{r}$ from their generators. There can be found several such methods in the literature $[4,8]$ but they all seem to require equicontinuity of $\left\{\alpha_{r}(\tau)\right\}_{\tau \in \boldsymbol{R}^{1}}$ in $\tau$ which we only know in the norm topology of $\mathscr{R}(\mathcal{O})$. Therefore we now require that $\alpha_{r}() A:. \boldsymbol{R}^{1}$ $\rightarrow \mathscr{B}(H)$ is continuous in the norm topology. It would be interesting to know if there exists any method not requiring equicontinuity and which reproduces $\alpha_{r}$ from its infinitesimal generator.

We proceed now to prove the existence of $\lim _{r \rightarrow \infty} \alpha_{r}$ for norm continuous $\alpha_{r}(.)^{7}$. It is natural to work with local concrete $C^{*}$-Algebras $\mathfrak{U}(\mathcal{O})$ in that case. Clearly the $\alpha_{r}(\tau)$ can be extended to the quasi-local algebra $\mathfrak{U}=\bigvee_{\mathcal{O}} \mathfrak{A}(\mathcal{O})^{8}$.

Theorem 2. Let $Q_{r}$ be e.s.a. on $D \subset H, \alpha_{r}(\tau) A=e^{i \tau Q_{r}^{*}} A e^{-i \tau Q_{r}^{*}} \in \mathfrak{A}$, $\forall A \in \bigcup_{\mathcal{O}} \mathfrak{A}(\mathcal{O})$

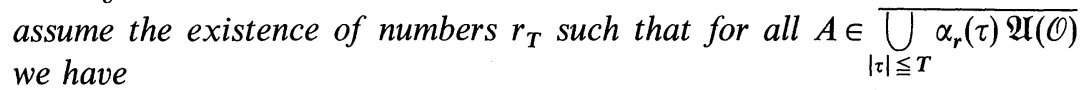

$$
\left(Q_{r} x, A y\right)-\left(x, A Q_{r} y\right)=\left(Q_{r^{\prime}} x, A y\right)-\left(x, A Q_{r^{\prime}} y\right), \quad \forall x, y \in D, r, r^{\prime} \geqq r_{T}
$$

and further the continuity of $\alpha_{r}() A:. \boldsymbol{R}^{1} \rightarrow \mathfrak{A}$ (in norm) then $\lim _{r \rightarrow \infty} \alpha_{r}(\tau)$ exists on $\mathfrak{A}, \forall \tau \in \boldsymbol{R}^{1}$.

Remark. Condition (3.1) expresses the fact that the symmetry belonging to $Q_{r}$ maps a bounded region $\mathcal{O}$ into some bounded region $\mathcal{O}_{T}$ if $|\tau| \leqq T$. Intuitively one would even expect that $\alpha_{r}(\tau) \mathfrak{A}(\mathcal{O}) \subset \mathfrak{A}\left(\mathcal{O}_{\tau}\right)$ ( $\tau$ fixed, $r$ sufficiently big) where $\mathcal{O}_{\tau}$ is the transformed region.

Before proving Theorem 2 we give a simple lemma on the resolvent of the generator of $\alpha_{r}($.$) .$

Lemma 5. Let $\alpha($.) be a continuous one-parameter group of contractions on a Banach space $X$ (i.e. $\|\alpha(\tau) x\| \leqq\|x\|, \forall x \in X, \tau \in \boldsymbol{R}^{1}$. If $\delta$ denotes $\left.\frac{d \alpha(\tau)}{d \tau}\right|_{\tau=0}$ the generator of $\alpha($.$) and R(z)=(z-\delta)^{-1}$ its resolvent then

$$
R(z)=\int_{0}^{T} e^{-z \tau} \alpha(\tau) d \tau+e^{-z T} \alpha(T) R(z), \quad \operatorname{Re} z>0 .
$$

\footnotetext{
${ }^{7}$ For a discussion of this norm-continuity see Ref. [9].

${ }^{8} \bigvee_{\mathcal{O}} \mathfrak{Q}(\mathcal{O})$ denotes the algebra generated by $\bigcup_{\mathcal{O}} \mathfrak{X}(\mathcal{O})$.
} 
Proof. We define $R_{T}(z)=\int_{0}^{T} e^{-z \tau} \alpha(\tau) d \tau$ then for $x \in X$

$$
\begin{aligned}
\alpha(t) R_{T}(z) x= & \int_{t}^{T+t} e^{-z(\tau-1)} \alpha(\tau) d \tau x \\
& \left.\Rightarrow \frac{d \alpha(t)}{d t} R_{T}(z) x\right|_{t=0}=\delta R_{T}(z) x=z R_{T}(z) x+e^{-z T} \alpha(T) x-x \\
& \Rightarrow R_{T}(z) x=R(z) x-e^{-z T} \alpha(T) R(z) .
\end{aligned}
$$

Proof of Theorem 2. Let $\delta_{r}$ denote the generator of $\alpha_{r}(),. R_{r}($.$) its$ resolvent. We want to show the existence of $\lim _{r \rightarrow \infty} R_{r}(z)$ on $\mathfrak{U}$ for $\operatorname{Re} z>0$. Assume $A \in \mathfrak{U}(\mathcal{O})$, then we may write

$$
\left(R_{r}(z)-R_{r^{\prime}}(z)\right) A=\delta_{r^{\prime}} R_{r^{\prime}}(z) R_{r}(z) A-R_{r^{\prime}}(z) \delta_{r} R_{r}(z) A \text { for } \operatorname{Re} z>0
$$

since $R_{r^{(\prime)}}(z) A$ lies in the domain of $\delta_{r^{(\prime)}}$. For $R_{r}(z)$ we use Lemma 5 to get $R_{r}(z)=\int_{0}^{T} e^{-z \tau} \alpha_{r}(\tau) d \tau+e^{-z T} \alpha_{r}(T) R_{r}(z)$.

Choosing $r$ and $r^{\prime} \geqq r_{T}$ and setting $A_{T}(z)=\int_{0}^{T} e^{-z \tau} \alpha_{r}(\tau) A d \tau$ we deduce from Eq. (3.1):

$$
\left|\left(Q_{r^{\prime}} x, A_{T}(z) y\right)\right| \leqq\|x\|\left(\left\|A_{T}(z) Q_{r}, y\right\|+\left\|\operatorname{ad} Q_{r}^{*} A_{T}(z) y\right\|\right), \quad \forall x, y \in D .
$$

That means $A_{T}(z) D \subset D\left(Q_{r^{*}}^{*}\right)$ and (again using (3.1) and Proposition 1)

We arrive at

$$
\delta_{r^{\prime}} A_{T}(z)=\left(\operatorname{ad} Q_{r^{\prime}}^{*} A_{T}(z)\right)^{-}=\left(\operatorname{ad} Q_{r}^{*} A_{T}(z)\right)^{-}=\delta_{r} A_{T}(z) .
$$

$\left(R_{r}(z)-R_{r^{\prime}}(z)\right) A=\delta_{r^{\prime}} R_{r^{\prime}}(z) e^{-z T} \alpha_{r}(T) R_{r}(z) A-R_{r^{\prime}}(z) \delta_{r} e^{-z T} R_{r}(z) \alpha_{r}(T) A$.

Using $\left\|\delta_{\left.r^{\prime}\right)} R_{r^{\prime}()}(z)\right\| \leqq 1,\left\|R_{r^{\prime}()}(z)\right\| \leqq \frac{1}{\operatorname{Re} z}$ we get $\left\|\left(R_{r^{\prime}}(z)-R_{r^{\prime}}(z)\right) A\right\|$ $\leqq e^{-T \operatorname{Re} z} \frac{2\|A\|}{\operatorname{Re} z}$ for $r, r^{\prime} \geqq r_{T}$. For $T \rightarrow \infty$ we get the existence of $\lim _{r \rightarrow \infty} R_{r}(z) A=R(z) A$ for $A \in \mathfrak{U}(\mathcal{O})$ from which the existence of the limit for all $A \in \mathfrak{A}$ follows by the equiboundedness of $R_{r}(z)$.

Next we want to show that the range of $R(z)$ is dense in $\mathfrak{A}$. For that we assume $A \in \mathfrak{A}(\mathcal{O})$ for bounded $\mathcal{O}$ then we get for $n \geqq 1$

$$
\begin{aligned}
\|n R(n) A-A\| \leqq\left\|\left(n R(n)-n R_{r}(n)\right) A\right\|+\left\|n R_{r}(n) A-A\right\| & \\
& \leqq 2 e^{-n T}\|A\|+\left\|n R_{r}(n) A-A\right\|
\end{aligned}
$$

for $r$ sufficiently big, which can be made arbitrarily small since $\lim _{n \rightarrow \infty} n R_{r}(n) A=A$ (see Ref. [4], p. 241). So we conclude $\lim _{n \rightarrow \infty} n R(n) A=A$ for all $A \in \mathfrak{U}(\mathcal{O})$. Since $\|n R(n)\| \leqq 1$ we get $\lim _{n \rightarrow \infty} n R(n) A=A$ for all $A \in \mathfrak{U}$. 
$R($.$) satisfying the resolvent equation R(z)-R\left(z^{\prime}\right)=\left(z^{\prime}-z\right) R(z) R\left(z^{\prime}\right)$ because the $R_{r}($.$) do, we can apply Lemma 1^{\prime}$ of Ref. [4], p. 217 which asserts that range $R(z)=\left\{A \in \mathfrak{A}: \lim _{n \rightarrow \infty} n R(n) A=A\right\}=\mathfrak{U}$.

Now we are prepared to apply the Trotter-Kato-Theorem Ref. [4], p. 269 on the convergence of semigroups proving the convergence of $\alpha_{r}(\tau)$ on $\mathfrak{A}$ for $\tau \geqq 0$. The proof for $\tau<0$ runs along the same lines. The limit $\alpha(\tau)=\lim _{n \rightarrow \infty} \alpha_{r}(\tau)$ is clearly a $C^{*}$-automorphism of $\mathfrak{A}$.

Finally we want to remark that the statements made above apply equally well to Quantum Statistical Mechanics, except time translations where condition (3.1) does not hold.

Acknowledgements. I greatly benefited from discussions with $\mathrm{H}$. Reeh who several times detected crucial errors in former versions of the proofs. I also want to thank S. Schlieder for a critical discussion of the subject.

\section{References}

1. Reeh, H.: Fortschr. Physik 16, 687 (1968).

2. Kastler, D.: Proceedings of the Rochester Conference on Particles and Fields, Rochester, N. Y. 1967.

3. c.f.e.g. Haag, R., u. D. Kastler: J. Math. Phys. 5, 848 (1964). - Borchers, H.J.: Commun. Math. Phys. 1, 57 (1965).

4. Yosida, K.: Functional analysis. Berlin-Heidelberg-New York: Springer 1965.

5. Kato, T.: Perturbation theory for linear operators. Berlin-Heidelberg-New York: 1966.

6. Streater, R. F.: Proceedings of the Conference on Mathematical Theory of Elementary Particles, ed. by. R. Goodman and I. Segal, MIT Press, 1966.

7. Moore, R. T.: Memoirs of the Am. Math. Soc. 78, 55-57 (1968).

8. Hille, E., and R. S. Phillips: Functional analysis and semi-groups. Amer. Math. Soc. Coll. Publ. Vol. XXXI, Providence, 1957.

9. Doplicher, S., D. Kastler, and D. W. Robinson: Commun. Math. Phys. 3, 1 (1966).

H. D. Maison

Max-Planck-Institut für Physik und Astrophysik 8000 München 23, Föhringer Ring 6 